\title{
A feasibility study on reduction of the entrance-surface dose to neonates by use of a new digital mobile X-ray system.
}

\section{AUTHOR(S):}

Utsunomiya, Satoru; Monzen, Hajime; Akimoto, Mami; Mukumoto, Nobutaka; Ishihara, Yoshitomo; Shiinoki, Takehiko; Nakamura, Mitsuhiro; ... Sato, Sayaka; Matsuo, Satoru; Hiraoka, Masahiro

\section{CITATION:}

Utsunomiya, Satoru ... [et al]. A feasibility study on reduction of the entrance-surface dose to neonates by use of a new digital mobile X-ray system.. Radiological physics and technology 2013, 6(1): 157-161

\section{ISSUE DATE:}

2013-01

URL:

http://hdl.handle.net/2433/170399

\section{RIGHT:}

The final publication is available at www.springerlink.com; この論文は 出版社版でありません。引用の際には出版社版をご確認ご利用くださ $\iota_{\circ}$; This is not the published version. Please cite only the published version. 
Authors:

Satoru Utsunomiya $\mathrm{PhD}^{1}$, Hajime Monzen $\mathrm{PhD}^{1}$, Mami Akimoto $\mathrm{MS}^{1}$,

Nobutaka Mukumoto MS ${ }^{1}$, Yoshitomo Ishihara MS ${ }^{1}$, Takehiko Shiinoki $\mathrm{PhD}^{1}$,

Mitsuhiro Nakamura $\mathrm{PhD}^{1}$, Yuki Miyabe $\mathrm{MS}^{1}$, Sayaka Sato $\mathrm{MS}^{1}$, Satoru Matsuo $\mathrm{PhD}^{2}$

and Masahiro Hiraoka MD, $\mathrm{PhD}^{1}$.

Title:

A feasibility study on reduction of the entrance surface dose to neonates by use of a new digital mobile $\mathrm{x}$-ray system

Affiliations and addresses of the authors:

${ }^{1}$ Department of Radiation Oncology and Image-Applied Therapy, Kyoto University

Graduate School of Medicine, Kyoto 606-8507, Japan

${ }^{2}$ Department of Radiology, Shiga Medical University Hospital, Shiga 520-2921, Japan

Corresponding Author:

All correspondence concerning this paper should be addressed to:

Hajime Monzen, Ph.D.

Department of Radiation Oncology and Image-Applied Therapy

Kyoto University Graduate School of Medicine

54 Shogoin-Kawaracho, Sakyoku, Kyoto 606-8507, Japan

Phone ${ }^{+}$81-75-751-3762, Fax: ${ }^{+}$81-75-771-9749

Email: hmon@kuhp.kyoto-u.ac.jp 


\begin{abstract}
:
We investigated the neonatal entrance-surface dose (ESD) and doses of scattered radiation emitted by a digital mobile X-ray system. The system is equipped with a novel flat-panel detector and is used in the neonatal intensive care unit. In the present study, the following three experiments were performed on frequently used X-ray-imaging condition: (1) the digital characteristics of the FPD were evaluated, (2) the ESD to a water-equivalent phantom was measured with a patient skin dosimeter (PSD), and (3) the scattered radiation around the incubator was measured with an ionization chamber survey meter. The digital characteristic curve showed that the system had excellent linearity and that the contrast characteristics were not affected by the tube voltage in the range of 50-110 $\mathrm{kV}$. The ESD was 51-52 $\mu \mathrm{Gy}$ with an 8-cm thick phantom and 33-34 $\mu \mathrm{Gy}$ with a $4.5-\mathrm{cm}$ phantom, for one exposure. The doses measured around the incubator were 0.1 to $0.6 \mu \mathrm{Sv}$ or below measurable limits. Use of the new device demonstrates the potential of reducing the ESD to the patient and operator.
\end{abstract}

Keywords:

entrance-surface dose, digital mobile X-ray system, flat-panel detector, neonatal intensive care unit 


\section{Introduction}

Chest X-ray examination is important for diagnosis of neonatal pulmonary disease. Considering the risk of carcinogenesis induced by X-ray exposure, the dose to which neonatal patients are exposed in such an examination should be kept as low as possible [1]. However, as frequent examinations are usually required, reducing the exposure is difficult.

It is well known that (compared with the ordinary film-screen system) the digital mobile X-ray imaging system (abbreviated in this article as "DMXS") equipped with a flat-panel detector (FPD) has the advantages of instantaneous image display, better sensitivity, and better spatial resolution [2]. Some new FPDs have been developed recently that reportedly have provided good image quality [3]. Therefore, the use of FPD-mounted X-ray examination systems, such as the DMXS, in the neonatal intensive care unit (NICU) or emergency room (ER), is spreading quickly throughout the world. The MobileDaRt Evolution (SHIMADZU Corporation, Kyoto) is a compactly designed DMXS equipped with the new CXDI-60C FPD (CANON INC., Tokyo) and is suitable for use in the NICU. By using the MobileDaRt Evolution in conjunction with the new infant incubator Incu i, Atom Infant Incubator 101 (ATOM MEDICAL Corporation, Tokyo), we can perform X-ray examinations easily without opening the incubator except to position and immobilize the patient. That excellent feature allows silent performance of the examination in the NICU [4].

Our aim in the present study was to demonstrate the potential of the DMXS equipped with a CXDI-60C in reducing the exposure of neonatal patients and of X-ray-system operators to X-rays in chest X-ray examinations. For this purpose, we examined the digital characteristics of the FPD for different tube voltages to evaluate the contrast characteristics. Next, we measured the entrance-surface dose (ESD) with a patient skin dosimeter (PSD) and a neonate-mimicking water-equivalent phantom placed in the NICU incubator (an Incu i, Atom Infant Incubator 101, ATOM MEDICAL Corp.). We also measured the exposure around the incubator by using an ionization chamber survey meter to estimate the exposure of a virtual operator or a person holding the patient during the examination.

2. Materials And Methods

\subsection{Equipment}


The CXDI-60C was used with the DMXS, called MobileDaRt Evolution. This FPD is an indirect detection system (outer dimensions $34.4 \mathrm{~cm}$ [W] x $38.0 \mathrm{~cm}$ [L] x $2.25 \mathrm{~cm}[\mathrm{H}])$ and has a high-sensitivity cesium iodide (CsI) scintillator. The total number of pixels is $1464 \times 1776$ (approximately $2.6 \times 10^{6}$ pixels); the pixel size is 0.16 $\mathrm{mm} \times 0.16 \mathrm{~mm}$, and the maximum image size is $23 \times 28 \mathrm{~cm}^{2}$. The density resolution is 4096 (12 bits). We also used the Incu i, Atom Infant Incubator 101 (outer dimensions 68 $\mathrm{cm}[\mathrm{W}]$ x $107 \mathrm{~cm}$ [L] x 138-178 cm [H]), an Unfors PSD (Unfors Instrument Incorporated, Billdal) with four detectors to measure dose, and a 451B-DE-SI (FLUKEBiomedical, Everett) ionization chamber survey meter.

\subsection{Digital characteristics of the DMXS}

To determine the digital characteristics of the DMXS, we measured exposure dose $15 \mathrm{~cm}$ away from the FPD facing toward the X-ray tube in order to remove the back-scatter component from the exposure dose [5]. Average readings of four detectors were taken, and the pixel value of images for tube voltages ranging from $50 \mathrm{kV}$ to 110 $\mathrm{kV}$ at $10 \mathrm{kV}$ intervals were obtained. The focus-film distance was set at $90 \mathrm{~cm}$, and the REX number [6] was fixed without adjustment of the density resolution or the performance of frequency processing. We obtained the average pixel value in the $100 \mathrm{x}$ 100 pixel area around the central axis for a given tube voltage. The current-time product (in mAs) was also varied from 0.32 to 3.2. The slope of the digital characteristic curve determined the contrast characteristics of the image for various tube voltages.

\subsection{ESD to neonate}

The current-time product was fixed at $0.8 \mathrm{mAs}$ as a frequently used X-ray imaging condition for neonate chest $\mathrm{X}$-ray imaging examination during the measurement by PSD of the ESD to the neonate-mimicking water-equivalent phantom. The measured ESD includes a back-scatter component from the phantom [7]. It is well known that the ESD increases as the phantom thickness increases for a fixed currenttime product. We performed measurements with the five different phantom thicknesses $(4.5,5.0,6.0,7.0$ and $8.0 \mathrm{~cm}$, corresponding to birth weights of 750,1000, 1500, 2000, $2500 \mathrm{~g}$, respectively), and the tube voltage was also varied $(53,54,56,58$ and $60 \mathrm{kV})$. The field size was fixed at $12 \mathrm{~cm} \times 12 \mathrm{~cm}$ or $19.5 \mathrm{~cm} \times 12.5 \mathrm{~cm}$ according to a previous report [7]. The focus-film distance was fixed at $90 \mathrm{~cm}$ as a frequently used X-rayimaging condition [7]. 


\subsection{X-ray-system operator exposure}

We measured radiation doses around the incubator by using an ionization chamber survey meter. The $8.0 \mathrm{~cm}$ thickness of the water-equivalent phantom corresponded to the maximum neonatal thickness. We performed the measurements with realistic parameter settings, namely, a field size of $12 \mathrm{~cm}$ x $12 \mathrm{~cm}$, a current-time product of $0.8 \mathrm{mAs}$, and a tube voltage of $60 \mathrm{kV}$ as selected for ESD measurement at the $8-\mathrm{cm}$ (maximum) thickness of the phantom. The phantom size was $30 \mathrm{~cm}$ [L] x 30 $\mathrm{cm}[\mathrm{R}]$. The setting for the direction exactly opposite the DMXS was 0 degrees, and the settings for the other directions were 45, 90, 135, 180, 225, and 315 degrees clockwise from the top. We measured the exposure for each direction. The distances from the central axis of the field to the point of measurement were 30,45 , and $60 \mathrm{~cm}$. The levels of measurement were approximately $100 \mathrm{~cm}$ and $130 \mathrm{~cm}$ from the ground.

\section{Results}

The digital characteristics of the DMXS for increasing tube voltages are presented in Fig. 1. The horizontal axis is the exposure dose on a logarithmic scale, measured by the PSD. The vertical axis is the pixel values of the image obtained. The current-time product was also varied from 0.32 to $3.2 \mathrm{mAs}$, and the values for each measurement are shown in the Fig. 1. The symbols in the figure indicate different values of X-ray tube voltages. As shown in the figure, the slope of the plotted lines which represent the contrast characteristics of the image is not affected much by the tube voltage. The higher the tube voltage, the larger the pixel value is for a fixed current-time product. The figure shows that the maximum pixel value was around 3700 . Considering that the tube voltage and the current-time product affect the level of exposure, we measured the ESD to neonates and the exposure dose around the incubator, assuming that the realistic settings were from 53 to $60 \mathrm{kV}$ (tube voltage) and $0.8 \mathrm{mAs}$ (currenttime product) [8] in the following measurements.

The measured ESD to neonates as a function of phantom thickness is presented in Fig. 2. The horizontal axis is the phantom thickness, and the vertical axis is the ESD measured at the phantom surface. The figure shows that the ESD varied 2-3\% between the two different field sizes: $12 \mathrm{~cm}$ x $12 \mathrm{~cm}$ and $19.5 \mathrm{~cm} \times 12.5 \mathrm{~cm}$. The ESD was 51$52 \mu \mathrm{Gy}$ at the maximum thickness $(8.0 \mathrm{~cm})$ of the phantom and 33-34 $\mu \mathrm{Gy}$ for the minimum thickness $(4.5 \mathrm{~cm})$ for one X-ray examination. 
The schematic diagram in Fig. 3 shows the points around which the incubator radiation dose was measured. The maximum dose was $0.6 \mu \mathrm{Sv}$ at point $\mathrm{A} 1(30 \mathrm{~cm})$, approximately $130 \mathrm{~cm}$ above the ground and facing directly opposite to the DMXS. For all other points, the dose was $0.1-0.3 \mu \mathrm{Sv}$, or no readings were obtained (Table 1). In Table 1 , the radiation doses in $\mu \mathrm{Sv}$ measured with an ionization chamber survey meter around the incubator are presented. The distances and levels (numbers in brackets) of the points of measurement are shown in the table. The locations of the points of measurement are shown in Fig. 3.

\section{Discussion}

Figure 1 shows that the DMXS equipped with a new FPD had an excellent linearity of the digital characteristics at $50-110 \mathrm{kV}$. The linearity of the system appears on a logarithmic scale for the exposure dose. Because around $0.8 \mathrm{mAs}$ is frequently used as a typical X-ray-imaging condition in the NICU [8], we did not evaluate the linearity for far lower current-time product (mAs) and corresponding pixel value. The linearity in the lower exposure dose region has been shown in some previous studies $[9,10,11]$, including previous models in the CXDI flat-panel detector series.

Figure 1 also shows that the contrast characteristics are not affected much by a change in tube voltage. For neonatal chest X-ray examination, a low tube voltage is favored for obtaining high-contrast images because the neonatal chest does not contain much air [12, 13]. We decided to use $53-60 \mathrm{kV}$ for the tube voltage for measurement of the exposure at different phantom thicknesses [7]. Figure 2 demonstrates that the exposure dose becomes greater as the phantom thickness increases. The reason is that back-scatter contributes to the increase as the phantom thickness increases. Therefore, we used the maximum thickness of the water-equivalent phantom $(8.0 \mathrm{~cm})$, a tube voltage $60 \mathrm{kV}$, and a current-time product $0.8 \mathrm{mAs}$ to estimate the exposure dose to which a neonate and the X-ray system operator were exposed.

Previous estimates of the ESD were 17-34 $\mu \mathrm{Gy}$ measured in the phantom of a newborn [7], $33 \mu \mathrm{Gy}$ (median dose; range : 3-75 $\mu \mathrm{Gy}$ ) for 1000-2500 g infants [8], and $70 \mu \mathrm{Gy}$ for a $10 \times 12 \mathrm{~cm}^{2}$ field size [14]. Our results showed lower or similar ESDs compared with previous studies. However, the examination conditions used in our studies were not optimized carefully in order to minimize the ESD. The result demonstrates the potential of the DMXS equipped with a CXDI-60C FPD in reducing the exposure of neonatal patients and X-ray system operators to X-rays in chest X-ray examinations. Except at one point of measurement, Table 1 shows that the ESD to an 
X-ray-system operator is very small $(<1 \mu \mathrm{Sv})$, or almost zero. The prominent maximum radiation dose was measured at point $\mathrm{A} 1(30 \mathrm{~cm}$ distance and $130 \mathrm{~cm}$ level, see Figure 3). This directional dependence might likely depend on positional relation between the DMXS and the incubator. Therefore, it is beyond the scope of this paper to investigate the reason for the directional dependence. The dose reported by Duetting et al. was higher, namely, less than $0.5 \mu \mathrm{Sv}$ at $30 \mathrm{~cm}$ away from the central beam at 150 $\mathrm{cm}$ vertical distance from the ground, than our estimate measured with the central beam in a similar position [14]. Because we did not carefully optimize the image quality to find the minimum exposure dose to a patient, the doses to which neonates and operators are exposed may be excessive, and it is very likely that these doses can be lowered further.

Finally, we conclude that the new DMXS may have the potential to lower the exposure compared to the conventional system, suggesting that the benefits of using the new system in the NICU, ER, operating room, or hospital room (which is usually not shielded) may be considerable. Possibly, X-ray-system operators or their aides could work without wearing protective clothing, especially when the patient is within an Incu i, Atom Infant Incubator 101. We showed that the radiation dose around the incubator from the DMXS is negligibly small. A particular advantage in the case of an emergency is that all staff can work without protection and thereby increase the workflow in a clinic.

\section{Conclusion}

In this study, we investigated the digital characteristics of the DMXS equipped with a new FPD for different tube voltages, and we showed that it has an excellent linearity of the digital characteristics and constant contrast characteristics for tube voltages in the range of 50-110 kV. The system can be used for significantly reducing the ESD to neonatal patients (examined by chest X-ray in the NICU) as well as the Xray system operator exposure more effectively than is available with conventional systems.

\section{ACKNOWLEDGMENT}

We would like to thank Mr. Masaaki Shibata and Mr. Masao Iinuma (engineers at SHIMADZU Corporation), who provided us with a laboratory where all of the 
measurements presented in this article were performed, and who assisted us during the work.

\section{REFERENCES}

1. International Commission on Radiological Protection. Radiological Protection and Safety in Medicine, ICRP Publication 73. Ann. ICRP 26 1996;1-47 (Oxford: Elsevier Science Ltd) .

2. Rong XJ, Shaw CC, Liu X, Lemacks MR and Thompson SK. Comparison of an amorphous silicon/cesium iodide flat-panel digital chest radiography system with screen/film and computed radiography systems--a contrast-detail phantom study. Med Phys. 2001;28:328-35.

3. Cowen AR, Kengyelics SM and Davies AG. Solid-state, flat-panel, digital radiography detectors and their physical imaging characteristics. Clin Radiol. 2008;87-98 .

4. Mutch SJ and Wentworth SD. Imaging the neonate in the incubator: an investigation of the technical, radiological and nursing issues. Br J Radiol. 2007;80:902-10.

5. Kishimoto K. Input-output Characteristics. Japanese Journal of Radiological Technology. 2009;65:985-991.

6. Shepard SJ, Wang J, Flynn M, Gingold E, Goldman L, Krugh K, Leong DL, Mah E, Ogden K, Peck D et al. An exposure indicator for digital radiography: AAPM Task Group 116 (executive summary). Med Phys. 2009;36:2898-914.

7. Ono K, Akahane K, Aota T, Hada M, Takano Y, Kai M and Kusama T. Neonatal doses from $\mathrm{X}$ ray examinations by birth weight in a neonatal intensive care unit. Radiat Prot Dosimetry. 2003;103:155-62.

8. Smans K, Struelens L, Smet M, Bosmans H and Vanhavere F. Patient dose in neonatal units. Radiat Prot Dosimetry. 2008;131:143-7.

9. Sakai S, Katayama R, Morishita J, Sakaguchi T, Ohkubo S, Maeda T and Hayabuchi N. Comparison of basic imaging properties for digital radiographic systems based on a direct conversion type of flat panel detector. Japanese Journal of Radiological Technology. 2008;64:41-9.

10. Katayama R. Actual measurements conditions of the basic imaging properties for digital radiographic systems. Japanese Journal of Radiological Technology. 2009;65:381-90.

11. Sato M, Eguchi Y, Yamada K, Kaga Y, Endo Y, Yamazaki T. Evaluation of a Flatpanel Detector System, Japanese Journal of Radiological Technology. 2001;57:68- 
77.

12. De Crop A, Bacher K, Van Hoof T, Smeets PV, Smet BS, Vergauwen M, Kiendys U, Duyck P, Verstraete K, D'Herde K et al. Correlation of contrast-detail analysis and clinical image quality assessment in chest radiography with a human cadaver study. Radiology. 2012;262:298-304.

13. Gislason AJ, Davies AG and Cowen AR. Dose optimization in pediatric cardiac Xray imaging. Med Phys. 2010;37:5258-69.

14. Duetting T, Foerste B, Knoch T, Darge K and Troeger. J. Radiation exposure during chest X-ray examinations in a premature intensive care unit: phantom studies. Pediatr Radiol. 1999;29:158-62. 
Table 1 Radiation dose in $\mu$ Sv measured with an ionization chamber survey meter around the incubator. The levels of measurement were approximately $100 \mathrm{~cm}$ and 130 $\mathrm{cm}$ from the ground. The symbols A1-3, C1-4, and E1-2 stand for the locations of the measurements shown in the Fig. 3. We performed the measurements with a field size of $12 \mathrm{~cm} \times 12 \mathrm{~cm}$, a current-time product $0.8 \mathrm{mAs}$, and a tube voltage $60 \mathrm{kV}$ as selected for ESD measurement at the 8-cm (maximum) thickness of the phantom.

\begin{tabular}{|c|c|c|c|c|c|c|c|c|}
\hline Level & A1 & $\mathrm{A} 2$ & $\mathrm{C} 1$ & $\mathrm{C} 2$ & $\mathrm{C} 3$ & $\mathrm{C} 4$ & $\mathrm{E} 1$ & E2 \\
\hline from & $(30$ & $(30$ & $(45$ & $(45$ & $(45$ & $(45$ & $(60$ & $(40$ \\
\hline ground & $\mathrm{cm})$ & $\mathrm{cm})$ & $\mathrm{cm})$ & $\mathrm{cm})$ & $\mathrm{cm})$ & $\mathrm{cm})$ & $\mathrm{cm})$ & $\mathrm{cm})$ \\
\hline $100 \mathrm{~cm}$ & 0 & 0.1 & 0 & 0 & 0 & 0.1 & 0 & 0.1 \\
\hline $130 \mathrm{~cm}$ & 0.6 & 0.1 & 0 & 0.1 & 0.1 & 0.3 & 0 & 0.1 \\
\hline
\end{tabular}


Fig. 1 Digital characteristics of the DMXS. The horizontal axis is the exposure dose measured by the PSD. The vertical axis is the pixel values of the image obtained. The current-time product was varied from 0.32 to $3.6 \mathrm{mAs}$, and the values for each measurement are shown near the symbols. The symbols in the figure indicate different values of X-ray tube voltages.

Fig. 2 Relationship between phantom thickness and exposure dose for $12 \mathrm{~cm} \mathrm{x} 12 \mathrm{~cm}$ and $19.5 \mathrm{~cm} \times 12.5 \mathrm{~cm}$ field sizes. The horizontal axis is the phantom thickness, and the vertical axis is the ESD measured at the phantom surface. The current-time product was fixed at $0.8 \mathrm{mAs}$ as a frequently used X-ray imaging condition for neonate chest X-ray imaging examination. We performed the measurements with five different phantom thicknesses $(4.5,5.0,6.0,7.0,8.0 \mathrm{~cm}$, corresponding to birth weights of 750, 1000, 1500,2000 and $2500 \mathrm{~g}$, respectively). The tube voltage (in $\mathrm{kV}$ ) was also varied (53, 54, $56,58,60)$. The focus-film distance was set at $90 \mathrm{~cm}$.

Fig. 3 Schematic diagram showing the points at which radiation exposures around the incubator Incu i, Atom Infant Incubator 101 (ATOM MEDICAL Corp.) and the DMXS, MobileDaRt Evolution (SHIMADZU Corporation) were measured. The numbers in parentheses are the distances from the central axis of the field to the point of measurement. 


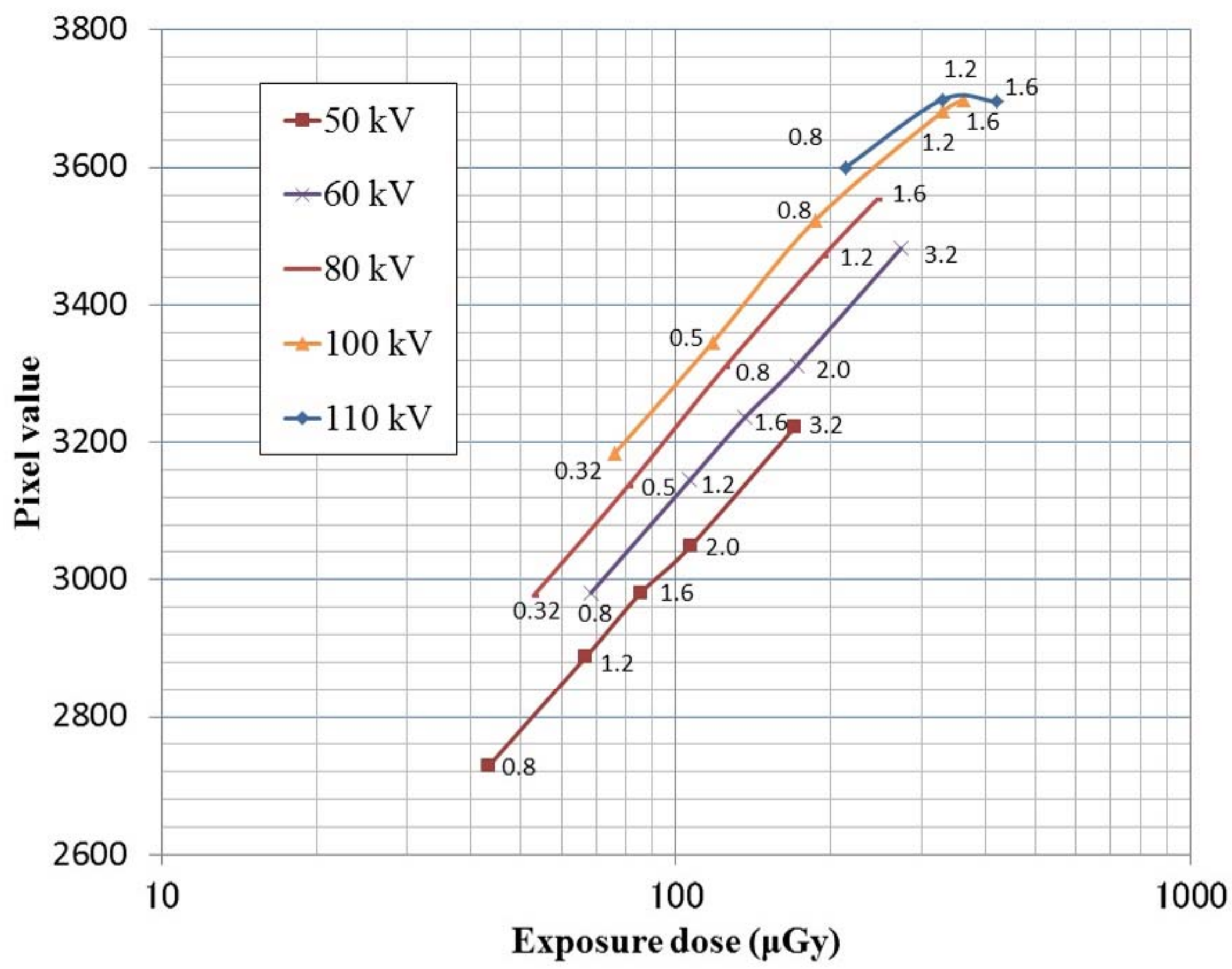

Fig. 1 


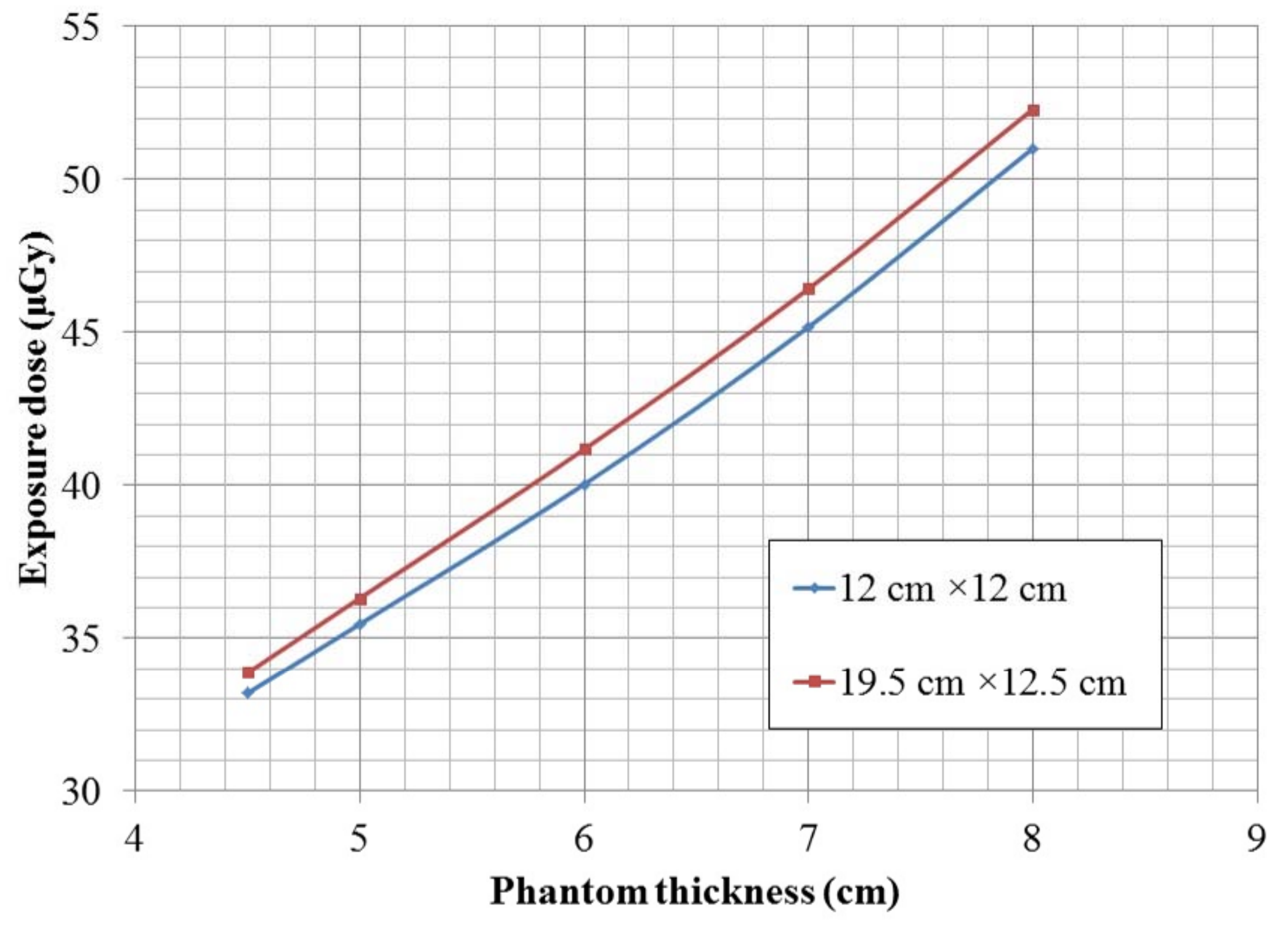

Fig. 2 


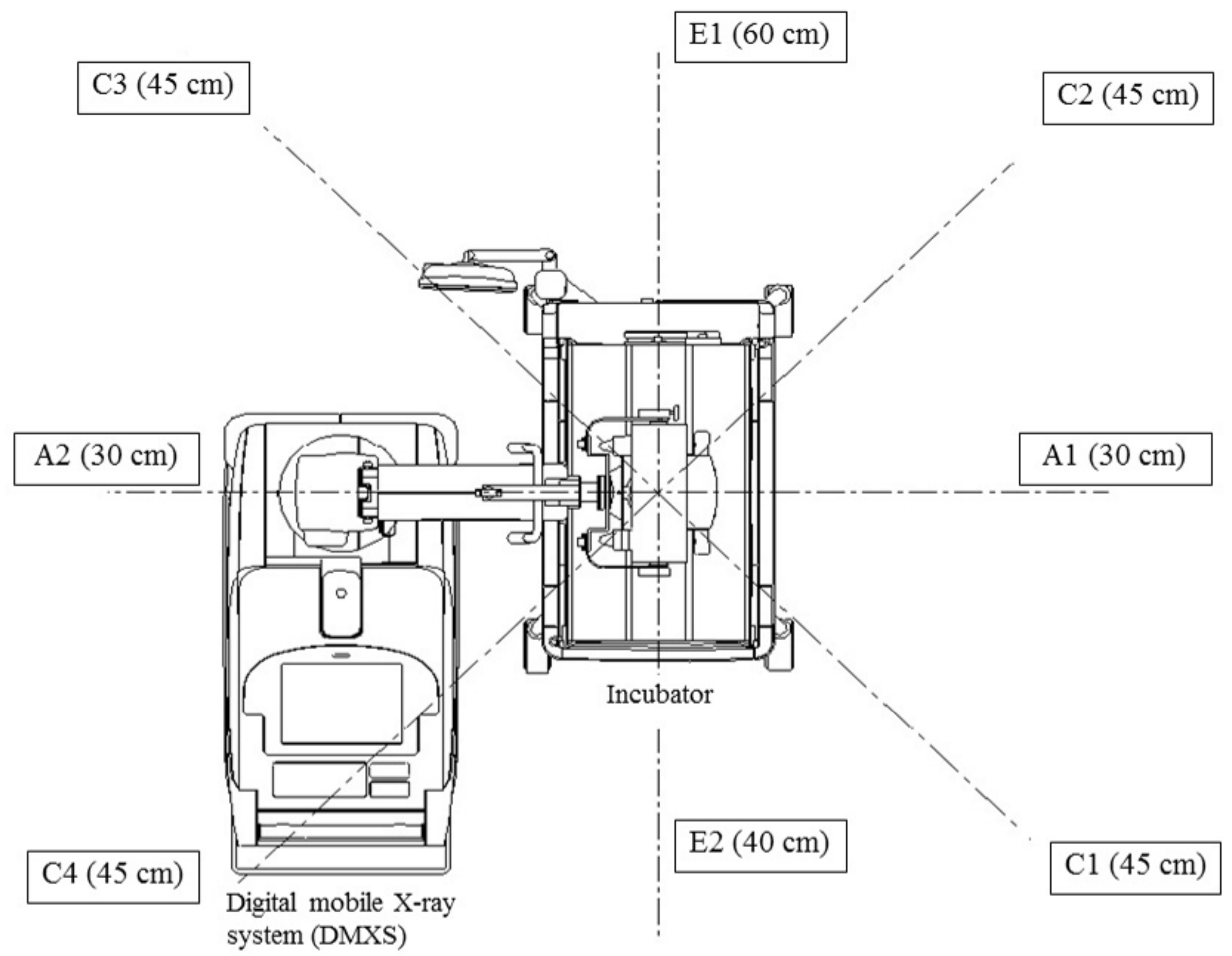

Fig. 3 